\title{
Modelos GAMLSS para analizar el grado secado de calcio dihidratado
} \author{
(9) $\Theta \Theta \Theta$ \\ Revista EIA \\ ISSN 1794-1237 \\ e-ISSN 2463-0950 \\ Año XVIII/ Volumen 18/ Edición N.35 \\ Enero-Junio de 2021 \\ Reia35014 pp. 1-13 \\ Publicación científica semestral \\ Universidad EIA, Envigado, Colombia
}

\section{Para citar este artículo /} To RefERENCE THIS ARTICLE / Hernández Barajas, F.; Cano Urrego, B.; Caicedo Chamorro, E.A. (2021). Modelos GAMLSS para analizar el grado secado de calcio dihidratado. Revista EIA, 18(35), Reia35014. pp. 1-13.

https://doi.org/10.24050/reia. v18i35.1439

\section{Autor de correspondencia:}

Hernández Barajas, F. (Freddy): ingeniero industrial, doctor en estadística, profesor asistente Universidad Nacional de Colombia sede Medellín.

Correo electrónico:

fhernanb@unal.edu.co

Recibido: 11- 06-2020

Aceptado: 13-12-2020

Disponible online: 19-01-2021

\author{
FREDDY HERNÁNDEZ BARAJAS ${ }^{1}$ \\ BRAHIAN CANO URREGO ${ }^{1}$ \\ EDWIN ALEXANDER CAICEDO CHAMORRO ${ }^{1}$
}

1. Universidad Nacional de Colombia sede Medellín.

\section{Resumen}

Transportar y secar materiales son dos actividades usuales en diferentes procesos productivos y los tornillos transportadores sirven para realizar estas dos actividades simultáneamente. Las condiciones de operación de un tornillo transportador son importantes porque influyen directamente en características finales como son el grado de secado del producto y la eficiencia térmica del proceso. En este artículo se emplearon los modelos GAMLSS para estudiar la influencia de tres variables operacionales, velocidad rotacional del tornillo, temperatura y flujo de alimentación, sobre el grado de secado del acetato de calcio dihidratado al ser transportado y secado en un tornillo. En este artículo se encontró que el modelo de regresión beta permite explicar el grado de secado mejor que otros modelos de regresión como el normal, exponencial, gamma, Weibull y logit-normal. Adicionalmente se encontró que, la operación del tornillo a una temperatura de $45^{\circ} \mathrm{C}$, velocidad rotacional de $3 \mathrm{rpm}$ y una velocidad de flujo de $6 \mathrm{~L} / \mathrm{min}$ permiten obtener el máximo grado de secado 35,46\%.

Palabras Claves: : modelos de regresión, GAMLSS, distribución beta, secador de tornillo transportador.

\section{GAMLSS models to analyze the drying degree of calcium dihydrate}

\section{Abstract}

Transporting and drying materials are two common activities in different production processes, and the conveyor screws are used to carry out these two activities simultaneously. The operating conditions of a conveyor screw are important because they directly influence final characteristics such as the degree of drying of the product and the thermal efficiency of the process. In this article, GAMLSS models were used to study the influence of three operational variables, screw rotational speed, temperature and feed flow, on the degree of drying of cal- 
cium acetate dihydrate when transported and dried in a screw. In this article it was found that the beta regression model allows to explain the degree of drying better than other regression models such as normal, exponential, gamma, Weibull and logit-normal. Additionally, it was found that the operation of the screw at a temperature of $45^{\circ} \mathrm{C}$, a rotational speed of $3 \mathrm{rpm}$ and a flow rate of $6 \mathrm{~L} / \mathrm{min}$ allow to obtain the maximum degree of drying 35,46\%.

Key Words: fregression model, GAMLSS, beta distribution, screw conveyor dryer.

\section{Introducción}

Los transportadores en forma de tornillo tienen numerosas aplicaciones en industrias de sectores como agrícola, de alimentos y bebidas, plástico, farmacéutica, química, minera, petróleo, gas e industrias de la construcción (Jumah y Mujumdar, 1995). Estos dispositivos, además de transportar, pueden cumplir otra función simultánea que es la de secar productos que vienen en forma de partículas sólidas, polvos finos, grumosos, pegajosos y materiales fibrosos. La actividad de secar productos es uno de los procesos más intensivos en lo que se refiere al consumo energía en la industria (Farzad y Yagoobi, 2020).

El desempeño de un sistema de secado depende de variables como la temperatura, humedad, distribución de tamaños, friabilidad y fluidez, entre otras. Por esta razón, en la literatura científica se han reportado varios estudios en los cuales se analiza la influencia de algunas variables operacionales en el desempeño del proceso de secado. A continuación, se presentan varios estudios en los cuales se usaron diferentes metodologías para abordar el desempeño de diversos sistemas de secado en función de algunas variables operativas.

Taruna y Jindal (2002) estudiaron el grado de secado de un subproducto del residuo de la pulpa de tofu al usar como instrumento de secado un lecho en movimiento continuo de partículas inertes. En el experimento se consideraron cuatro variables operacionales que fueron masa de gránulos de teflón utilizados como partículas inertes, velocidad de alimentación, temperatura del aire de entrada y velocidad del flujo de aire. Utilizando un análisis de varianza los autores identificaron que el grado de secado del subproducto se incrementó con el aumento de la velocidad de alimentación y la masa de los gránulos de teflón.

Reyes et al. (2008) utilizaron un diseño factorial para estudiar la cinética del secado de aserrín cuando se usa un secador en lecho fluidizado. En el estudio usaron un diseño factorial $2^{3}$ en el cual variaron de la velocidad del aire caliente, la temperatura del aire caliente y la velocidad de giro de los platos. Usando análisis de varianza, los autores identificaron que como variables significativas en el proceso de secado de aserrín a la velocidad del aire y temperatura del aire.

Triana et al. (2009) analizaron el grado de secado del acetato de calcio dihidrato cuando pasa por un secador tipo tornillo transportador. Estos autores realizaron un experimento variando la temperatura, velocidad y flujo de alimentación en el secador. Usando un modelo de regresión lineal múltiple y superficies de respuesta, los autores identificaron que el punto óptimo con temperatura de $45^{\circ} \mathrm{C}$, flujo de $8 \mathrm{~L} / \mathrm{min}$ y velocidad de 3 rpm maximiza el grado de secado.

Jafari y Zare (2017) realizaron un experimento factorial para investigar la influencia del ultrasonido de alta potencia en el secado de arroz con lecho fluidizado con el objetivo de disminuir el contenido de humedad y el tiempo de secado del arroz. En este estudio los autores consideraron como factores de interés la potencia de ultrasonido, la frecuencia y la temperatura del aire de secado. Usando un análisis 
de varianza, los autores encontraron que todos los factores fueron significativos para explicar el contenido de humedad y el tiempo de secado del arroz.

Mustaffar et al. (2018) informaron sobre un nuevo tipo de secador de tornillo transportador con calentamiento pasivo por un tubo de calor anular en el secado de una suspensión de materia prima de cerámica. Los tres factores considerados fueron relación de llenado, inclinación y temperatura, todos en tres niveles para un diseño factorial $3^{3}$. Usando un análisis de varianza, los autores concluyeron que los efectos de la relación de llenado y la inclinación fueron significativos en reducir la humedad, mientras la temperatura no tuvo un efecto significativo.

Bai et al. (2018) realizaron un estudio en el cual se secaron de semillas de ginkgo biloba bajo diferentes potencias de microondas $(200,280,460$ y $640 \mathrm{~W})$ con el objetivo de estudiar propiedades de secado y cambios en el color de las semillas secas. Los autores utilizaron una red neuronal para predecir la humedad final y los parámetros de color de las semillas de ginkgo biloba. Los resultados mostraron que la metodología de redes neuronales podía predecir con precisión los datos experimentales con un alto coeficiente de correlación $(0,9056-0,9834)$ y un error cuadrado medio bajo (0,0014-2,2044).

Li et al. (2019) presentaron una revisión de la literatura de los últimos cinco años en la cual se identifican nuevas tecnologías (microondas, radiofrecuencia, radiación infrarroja y ultrasonido) para secar frutas y verduras. Uno de los aportes de este trabajo es la identificación de técnicas para modelar las características de secado y cambios de calidad de varios productos agrícolas. Entre estas técnicas se destacan metodología de superficie de respuesta, predicción con lógica difusa, regresión parcial de mínimos cuadrados, redes neuronales, entre otras.

Debido a que el secado de productos es una actividad importante en muchos procesos productivos, se hace necesario contar con metodologías que permitan predecir las características finales del producto luego del proceso de secado. Por esta razón, en el presente artículo se retoman los datos experimentales de Triana et al. (2009) y se vuelven a analizar utilizando modelos GAMLSS (generalized additive models for location, scale and shape) con el objetivo de encontrar un nuevo modelo para explicar mejor el desempeño del proceso de secado en función de las variables operaciones.

El artículo está estructurado de la siguiente forma. En la Sección 2.1 se presenta una descripción breve del experimento y de los datos utilizados. En la Sección 2.2 se hace una presentación de los modelos GAMLSS. En la sección 2.3 se explica el procedimiento utilizado para seleccionar las distribuciones estadísticas de la variable respuesta en los modelos de regresión. En la Sección 3 se presentan los diferentes modelos ajustados para explicar la variable respuesta grado de secado en función de las variables operacionales. Finalmente, en la Sección 4 se presentan las conclusiones del artículo.

\section{Materiales y métodos}

\subsection{Descripción del experimento}

Triana et al. (2009) usaron un diseño factorial $2 \times 3^{2}$ para medir el porcentaje de grado de secado (GS) del acetato de calcio dihidratado mediante un secador de tipo transportador en función de las covariables: velocidad rotacional del tornillo (N) en dos niveles 3 y $7 \mathrm{rpm}$, la temperatura ( $T$ ) del sólido en tres niveles 45, 53, 61 grados 
centígrados y el flujo de alimentación $(C)$ en tres niveles de 6, 8 y $10 \mathrm{~L} / \mathrm{min}$. En la Figura 1 se muestra un esquema del secador tipo tornillo transportador usado en el experimento.

Los elementos que conforman el sistema de secado fueron: un cristalizador con chaqueta C-101, un tornillo secador S-101, una bomba de doble diafragma B-101 y válvulas de recirculación y bloqueo (V-102, V-101 y V-103). La presión del aire de entrada se mantuvo constante en 40 psi y el SCD usado tenía 8.66 metros de longitud y $0.17 \mathrm{~m}$ de diámetro del tornillo. La temperatura $\left[{ }^{\circ} \mathrm{C}\right]$ del tornillo secador se controló por medio de la válvula V-105. La variable velocidad del tornillo [rpm] se controló por medio del variador de frecuencia VG-101 y el flujo de alimentación [L/min] se controló con la válvula V-104.

\section{Figura 1. Ilustración sistema de secado usado en el experimento.} Fuente: Triana et al. (2009).

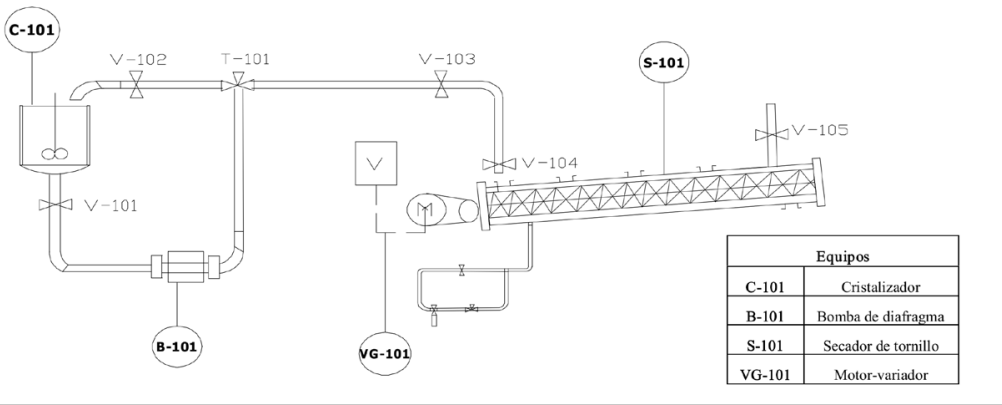

En la Tabla 1 se presentan los niveles de las variables de estudio $(T, N$ y $C$ ) y la variable respuesta (GS). Triana et al. (2009) decidieron eliminar las observaciones $1 \mathrm{y}$ 13 destacadas en negrita porque estos datos causan una desviación adimensional que se atribuye a una propiedad del sólido no controlada. Al igual que Triana et al. (2009), aquí se usaron solo las observaciones restantes para construir los modelos de regresión. 
Tabla 1. Datos del experimento obtenidos de Triana et al. (2009). Entre paréntesis está el nombre abreviado de la variable y dentro de corchetes las unidades de la variable.

\begin{tabular}{|c|c|c|c|c|}
\hline Observación & $\begin{array}{c}\text { Temperatura } \\
(T)\left[{ }^{\circ} \mathrm{C}\right]\end{array}$ & $\begin{array}{l}\text { Velocidad rotacional } \\
\text { del tornillo } \\
(N)[\mathrm{rpm}]\end{array}$ & $\begin{array}{c}\text { Flujo de } \\
\text { alimentación } \\
(C)[\mathrm{L} / \mathrm{min}]\end{array}$ & $\begin{array}{l}\text { Grado de } \\
\text { secado } \\
(G S)[\%]\end{array}$ \\
\hline 1 & 45 & 3 & 6 & 0,25 \\
\hline 2 & 45 & 3 & 8 & 12,08 \\
\hline 3 & 45 & 3 & 10 & 7,23 \\
\hline 4 & 53 & 3 & 6 & 5,80 \\
\hline 5 & 53 & 3 & 8 & 0,59 \\
\hline 6 & 53 & 3 & 10 & 1,17 \\
\hline 7 & 61 & 3 & 6 & 14,13 \\
\hline 8 & 61 & 3 & 8 & 5,94 \\
\hline 9 & 61 & 3 & 10 & 7,49 \\
\hline 10 & 45 & 7 & 6 & 2,09 \\
\hline 11 & 45 & 7 & 8 & 3,84 \\
\hline 12 & 45 & 7 & 10 & 1,39 \\
\hline 13 & 53 & 7 & 6 & 3,61 \\
\hline 14 & 53 & 7 & 8 & 0,22 \\
\hline 15 & 53 & 7 & 10 & 1,31 \\
\hline 16 & 61 & 7 & 6 & 5,75 \\
\hline 17 & 61 & 7 & 8 & 5,35 \\
\hline 18 & 61 & 7 & 10 & 9,58 \\
\hline
\end{tabular}

\subsection{GAMLSS (Generalized Additive Model for Location Scale and Shape)}

Los modelos de regresión son una de las herramientas más populares en estadística y sus orígenes se encuentran a finales del siglo XVIII. Legendre (1805) reportó el método de mínimos cuadrados y lo usó para analizar un conjunto de datos provenientes de una encuesta en la cual se tenían dos variables y cinco observaciones. Gauss (1809) publicó una justificación del método de mínimos cuadrados e introdujo la distribución normal para los errores del modelo.

En los modelos de regresión el objetivo es explicar la media (o valor esperado) de una variable y en función de un conjunto de $p$ variables explicativas $x_{1}, \cdots, x_{p}$ de la siguiente manera.

$$
\mu=E\left(y \mid x_{1}, \cdots, x_{p}\right)=\beta_{0}+\beta_{1} x_{1}+\cdots+\beta_{p} x_{p}
$$

Los supuestos de este modelo de regresión son:

- se disponen de la información de $p$ variables explicativas y una variable respuesta y para $n$ unidades muestrales o casos,

- las variables observadas para un caso son independientes de los otros casos, 
- la variable respuesta y debe tener distribución normal $N\left(\mu, \sigma^{2}\right)$ y

- la varianza $\sigma^{2}$ debe ser constante.

En la práctica los usuarios se encuentran con variables respuesta que no necesariamente tienen distribución normal, como el número de defectos en un sistema productivo (variable de conteo), el contenido neto de una bebida gaseosa (variable positiva) o el porcentaje de un medicamento que queda en el cuerpo de algún paciente luego de cierto periodo (variable entre 0 y 1 ). En estos casos sería un error usar un modelo de regresión clásico ya que se viola el supuesto de normalidad y por lo tanto se hace necesario constar una alternativa de modelación.

Los modelos GAMLSS (generalized additive models for location, shape and scale) fueron propuestos por Rigby y Stasinopoulos (2005) surgieron como una extensión a los modelos de regresión tradicionales porque flexibilizan el supuesto de normalidad de la variable respuesta y permiten que el usuario pueda elegir la distribución entre un listado de más de cien distribuciones. Otra ventaja de los modelos GAMLSS es que se pueden modelar todos los parámetros de la distribución seleccionada y no solo la media como en los modelos de regresión tradicionales.

Los modelos GAMLSS asumen que se dispone de $n$ observaciones independientes y con función de densidad (o de masa) $f(y \mid \theta)$ en la cual el vector $\theta=(\mu, \sigma, \eta, \tau)^{T}$ representa el vector parámetros que se pueden modelar en función de las variables explicativas. Los primeros dos parámetros $\mu$ y $\sigma$ corresponden por lo general a los parámetros de ubicación y escala de la distribución, mientras que los parámetros restantes (si aplican a la distribución) están asociados a otras características de la distribución.

Simbólicamente los modelos GAMLSS se puede escribir de la siguiente manera.

$$
\begin{aligned}
& g_{1}(\mu)=\beta_{10}+\beta_{11} x_{1}+\cdots+\beta_{1 p} x_{p}, \\
& g_{2}(\sigma)=\beta_{20}+\beta_{21} x_{1}+\cdots+\beta_{2 p} x_{p} \\
& g_{3}(v)=\beta_{30}+\beta_{31} x_{1}+\cdots+\beta_{3 p} x_{p} \\
& g_{4}(\tau)=\beta_{40}+\beta_{41} x_{1}+\cdots+\beta_{4 p} x_{p}
\end{aligned}
$$

donde las funciones $g_{\mathrm{k}}(\cdot)$ con $k=1,2,3,4$ se conocen como funciones de enlace y sirven para transformar el lado derecho (predictor lineal) de cada ecuación anterior en valores apropiados para cada parámetro. Los parámetros $\beta_{k j} \operatorname{con} j=0,1, \cdots, p$ corresponden a los efectos de las variables explicativas y se estiman por el método de máxima verosimilitud. Los modelos GAMLSS son muy flexibles porque en el lado derecho (predictor lineal) de las cuatro expresiones anteriores, se pueden incluir polinomios, polinomios fraccionales, splines, efectos aleatorios, entre otros.

En el lenguaje de programación R (2020) está disponible el paquete gamlss con el cual los usuarios pueden aplicar modelos GAMLSS de una forma sencilla. Este paquete cuenta con más de 100 distribuciones continuas, discretas y mixtas para seleccionar la distribución de la variable respuesta y es posible que el usuario defina su propia función de densidad, para una mayor compresión de estos modelos se recomienda consultar Stasinopoulos et al. (2017). 


\subsection{Selección de la distribución para la variable respuesta}

Para identificar las distribuciones de probabilidad (con soporte en $R, R^{+}$y $(0,1)$ ) que mejor explican el comportamiento de la variable porcentaje grado de secado, se usó un enfoque marginal, es decir, sin utilizar covariables auxiliares. Para esto se usó la función fitDist del paquete gamlss la cual suministra una lista de distribuciones que se ajustan mejor a la variable respuesta. El criterio de selección interno de fitDist es el GAIC (criterio de información de Akaike generalizado) propuesto por Akaike (1974).

En la Figura 2 se presenta seis veces el histograma de la variable respuesta grado de secado $(G S)$ y las curvas de densidad empíricas para las distribuciones normal, exponencial, Weibull 3, gamma, beta y logit-normal. Los valores de GAIC en esta búsqueda marginal para las distribuciones normal, exponencial, Weibull 3, gamma, beta y logit-normal fueron $-55,33 ;-61,32 ;-60,96 ;-60,71 ;-60,95$ у $-58,28$ respectivamente. Aunque la distribución normal tiene soporte en los números reales y la variable respuesta es positiva, aquí se incluyó la distribución normal como distribución de referencia ya que fue la usada por Triana et al. (2009) para analizar los datos experimentales.

Figura 2. Histograma para la variable grado de secado con las curvas de densidad empíricas para las distribuciones normal, exponencial, Weibull 3, gamma, beta y logit-normal.
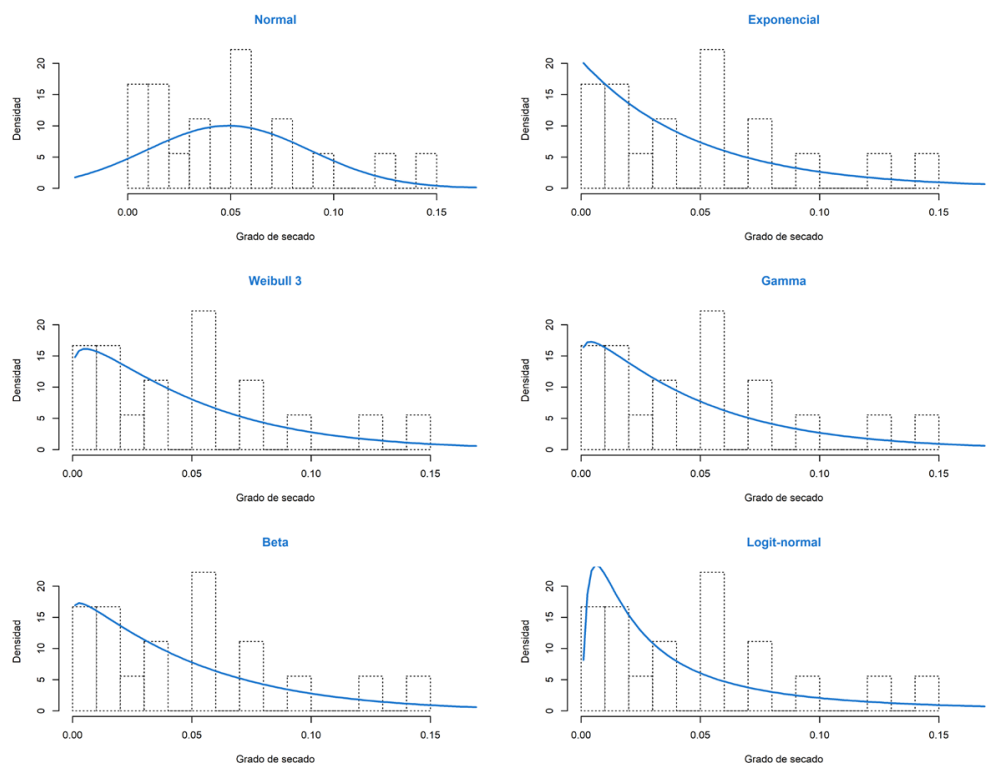

De la figura anterior se observa que las seis densidades (distribuciones) intentan reproducir la forma del histograma. De las seis distribuciones consideradas, la exponencial es la única con un parámetro, mientras que las restantes tienen dos parámetros. Estas seis distribuciones identificadas aquí fueron las distribuciones usadas para construir los modelos de regresión que se presentan en la siguiente sección. 


\section{Resultados}

\subsection{Aplicación de los modelos de regresión}

Triana et al. (2009) ajustaron un modelo de regresión lineal múltiple y usaron las variables velocidad rotacional del tornillo $(N)$, temperatura $(T)$ y flujo de alimentación $(C)$ para explicar el grado de secado $(G S)$. El modelo ajustado que reportaron Triana et al. (2009) se muestra a continuación:

$$
\begin{gathered}
\hat{E}(G S)=372,57-11,31 T-10,30 C-11,14 N+0,10 T^{2}+0,28 C^{2}+0,05 T \times C \\
+0,11 T \times N+0,54 C \times N
\end{gathered}
$$

Este modelo se caracteriza por tener un pseudo $R^{2}=0,86$ con una correlación lineal de Pearson entre las observaciones $y_{i}$ y las estimaciones $\hat{E}\left(y_{i}\right)$ de 0,93 . Este modelo se usará como modelo de referencia para comparar los modelos propuestos.

En el presente artículo se consideraron modelos GAMLSS con variable respuesta distribuida normal, exponencial, Weibull 3, gamma, beta y logit-normal. La distribución normal tiene soporte en R, la distribución exponencial, Weibull 3 y gamma tienen soporte en $\mathrm{R}^{+}$mientras que las distribuciones beta y logit-normal tienen soporte en $(0 ; 1)$.

Para cada uno de estos casos se modeló el parámetro $\mu$ de cada distribución en función de las variables velocidad rotacional del tornillo $(N)$, temperatura $(T)$ y flujo de alimentación $(C)$, usando términos cuadráticos e interacciones entre estas variables. Para las distribuciones normal, Weibull 3, gamma, beta y logit-normal que tienen un segundo parámetro $\sigma$, no se utilizaron covariables en la modelación de este parámetro debido a que sólo se cuentan con 16 observaciones experimentales lo cual dificulta la estimación de efectos.

En la Tabla 2 se presenta un resumen de los modelos ajustados. En la segunda fila de la Tabla 2 está el modelo de referencia propuesto por Triana et al. (2009) y en las filas siguientes están los modelos finales luego de un proceso de selección de variables. En las tres últimas columnas de la Tabla 2 se presentan tres medidas para evaluar y comparar los modelos. La columna de correlación corresponde al coeficiente de correlación lineal de Pearson entre las observaciones $y_{i}$ y las estimaciones $\hat{E}\left(y_{i}\right)$ dadas por el modelo ajustado.

\section{Tabla 2. Medidas de comparación para los modelos ajustados.}

\begin{tabular}{|c|c|c|c|c|c|}
\hline Modelo & Distribución & $\begin{array}{c}\text { Variable } \\
\text { respuesta }\end{array}$ & Correlación & GAIC & Pseudo $R^{2}$ \\
\hline Referencia & Normal & $G S$ & 0,93 & $-79,03$ & 0,86 \\
\hline Mod1 & Normal & $\sqrt{G S}$ & 0,92 & $-57,14$ & 0,90 \\
\hline Mod2 & Exponencial & $G S$ & 0,73 & $-66,65$ & 0,41 \\
\hline Mod3 & Gamma & $G S$ & 0,96 & $-82,84$ & 0,85 \\
\hline Mod5 & Weibull 3 & $G S$ & 0,96 & $-84,06$ & 0,86 \\
\hline Mod6 & Beta & $G S$ & 0,98 & $-94,86$ & 0,93 \\
\hline Mod7 & Logit-normal & $G S$ & 0,83 & $-75,16$ & 0,73 \\
\hline
\end{tabular}


Una característica deseable en un modelo es que genere estimaciones $\hat{E}\left(y_{i}\right)$ cercanas a los valores reales $\left(y_{i}\right)$ de la variable de interés. De la tabla anterior se observa que los modelos gamma, Weibull $3 \mathrm{y}$ beta presentaron los mayores valores de correlación y en particular el modelo beta presentó la más alta correlación con un valor de 0.98 .

En la Figura 3 se muestra el diagrama de dispersión entre $y_{i}$ y $\widehat{E}\left(y_{i}\right)$ para los modelos gamma, Weibull 3 y beta. La línea a $45^{\circ}$ que se observa en los diagramas de dispersión sirve como referencia para saber si las estimaciones son cercanas a los valores observados. De la figura se observa que las estimaciones $\widehat{E}\left(y_{i}\right)$ acompañan a los valores reales $y_{i}$ del grado de secado observados en el experimento.

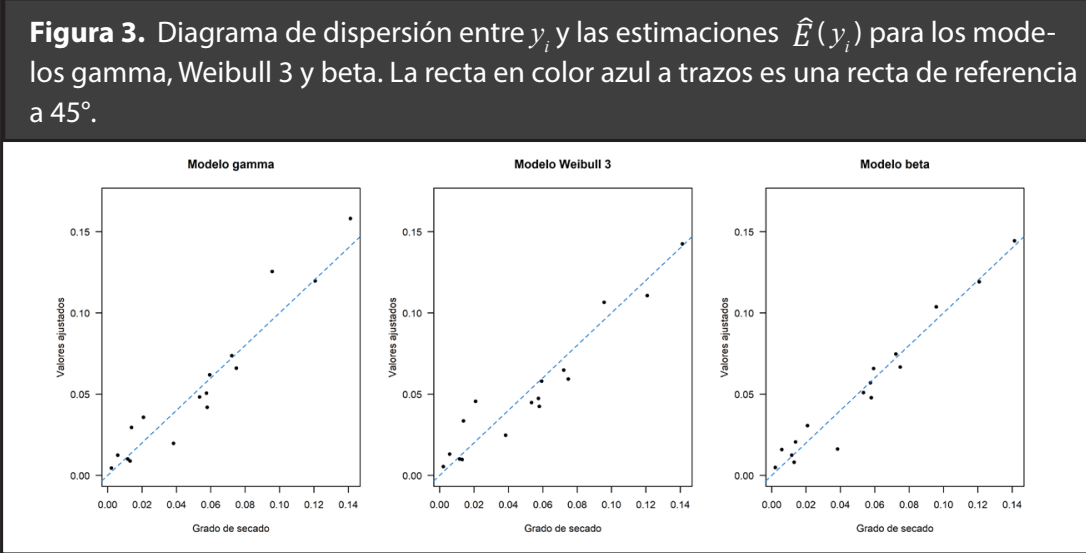

Los residuales RQR (randomized quantile residuals) son útiles para evaluar modelos cuya variable respuesta es diferente a la normal y éstos residuales tienen distribución normal si el modelo fue bien ajustado (Stasinopoulos et al., 2017). En la Figura 4 se muestran los gráficos cuantil-cuantil para los residuales RQR de los modelos gamma, Weibull 3 y beta. De la figura se observa que los residuales están, en la mayoría de los casos, dentro de las bandas de confianza de color azul lo que indica que los residuales tiene distribución normal estándar. Adicionalmente, se aplicó la prueba Kolmogorov-Smirnov para estudiar la normalidad de los residuales y se encontraron valores-P de 0,88; 0,50 y 0,90 para los residuales de los modelos gamma, Weibull 3 y beta respectivamente, lo cual indica que podemos asumir que los residuales tienen distribución normal estándar.

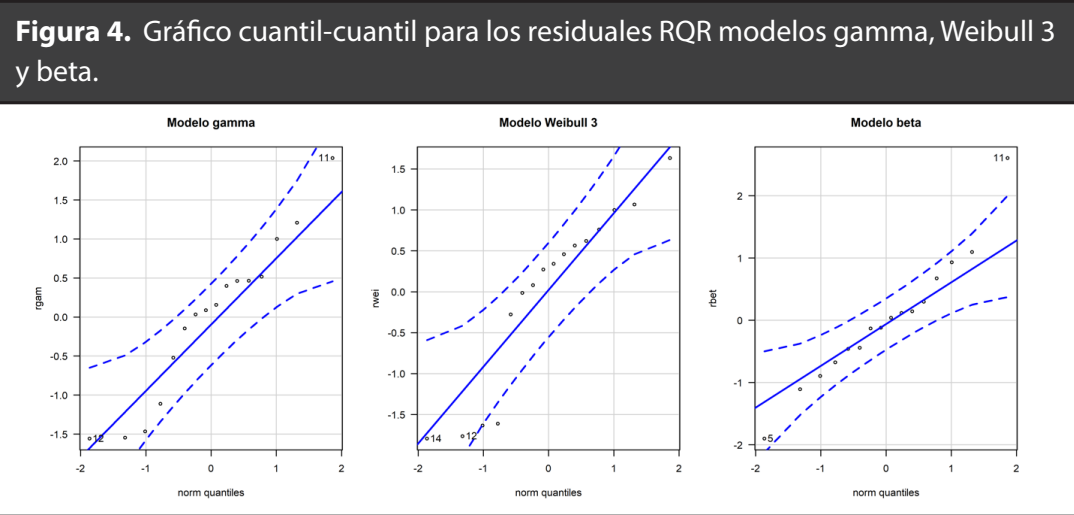


Al comparar los modelos según su GAIC, correlación, Pseudo $R^{2}$ y normalidad de los residuales, se decidió elegir el modelo beta como modelo final porque éste presentó los mejores resultados. En la siguiente tabla se presenta el resumen de los efectos estimados para el modelo beta.

\section{Tabla 3. Efectos estimados para el modelo con respuesta beta.}

\begin{tabular}{|c|r|c|c|c|}
\hline \multicolumn{5}{|c|}{ Efectos estimados en la modelación de logit $(\mu)$} \\
\hline & Estimación & Error estándar & Valor- $t$ & Valor- $P$ \\
\hline Intercepto & 101,72 & 10,76 & 9,45 & 0,00 \\
\hline$T$ & $-3,24$ & 0,34 & $-9,44$ & 0,00 \\
\hline$N$ & $-2,57$ & 0,32 & $-8,13$ & 0,00 \\
\hline$C$ & $-3,28$ & 073 & $-4,51$ & 0,00 \\
\hline$T^{2}$ & 0,03 & 0,00 & 9,20 & 0,00 \\
\hline$C^{2}$ & 0,11 & 0,03 & 3,25 & 0,01 \\
\hline$T \times N$ & 0,03 & 0,00 & 6,12 & 0,00 \\
\hline$N \times C$ & 0,09 & 0,02 & 4,83 & 0,00 \\
\hline$T \times C$ & 0,02 & 0,01 & 2,73 & 0,03 \\
\hline \multicolumn{7}{|r|}{ Efectos estimados en la modelación de logit $(\sigma)$} \\
\hline Intercepto & Estimación & Error estándar & Valor- $t$ & Valor- $P$ \\
\hline
\end{tabular}

Usando la información de la tabla anterior es posible escribir las expresiones matemáticas para los parámetros $\hat{\mu}$ y $\hat{\sigma}$ del modelo de regresión beta. En el modelo de regresión beta ambos parámetros $\mu$ y $\sigma$ deben estar en el intervalo $(0,1)$, es por esa razón es que se usa la función de enlace logit en las siguientes expresiones.

$$
\begin{aligned}
& \operatorname{logit}(\hat{\mu})= 101,72-3,24 T-2,57 N-3,28 C+0,03 T^{2}+0,11 C^{2}+0,03 T \times N \\
&+0,09 N \times C+0,02 T \times C \\
& \operatorname{logit}(\hat{\sigma})=-2,90
\end{aligned}
$$

Con la parametrización de la distribución beta usada en este artículo el valor esperado y la varianza están dadas por $E(X)=\mu$ y $\operatorname{Var}(X)=\sigma^{2} \mu(1-\mu)$ respectivamente. Esto significa que el parámetro $\mu$ coincide con el valor esperado de la variable y por lo tanto el valor estimado (promedio) de la variable GS se puede escribir así:

$$
\begin{aligned}
\hat{E}(G S)= & \hat{\mu}=\operatorname{logit}^{-1}\left(101,72-3,24 T-2,57 N-3,28 C+0,03 T^{2}+0,11 C^{2}\right. \\
& +0,03 T \times N+0,09 N \times C+0,02 T \times C)
\end{aligned}
$$

De igual forma la varianza estimada para la variable $G S$ se puede escribir en forma compacta (en términos de $\hat{\mu}$ y $\hat{\sigma}$ ) así:

$$
\widehat{\operatorname{Var}}(G S)=\hat{\sigma}^{2} \hat{\mu}(1-\hat{\mu})
$$


Usando la ecuación de $\widehat{E}(G S)$ mostrada arriba, se aplicó un método de búsqueda para obtener los valores de $T, N$ y $C$ que maximizan la variable grado de secado $G S$ y lo que se encontró fue que el valor máximo grado de secado es 35,46\% (o 0,3546 en fracción) cuando $T=45, N=3$ y $C=6$.

Como la expresión para $\hat{E}(G S)$ involucra tres variables, la variable temperatura (T) se fijó en sus cuantiles $Q_{1}=45, Q_{2}=53$ y $Q_{3}=61$ para poder generar los gráficos de contornos para $\widehat{E}(G S)$ mostrados en la Figura 5. En el panel izquierdo de la figura se observa un punto de color azul que corresponde a la combinación $T=45, N=3$ y $C=$ 6, la cual maximiza el grado de secado a un valor de 35,46\% (o 0,3546 en fracción).

Figura 5. Gráfico de contornos para $\widehat{E}$ (GS). En la izquierda el gráfico de contornos para $T=45$, en la mitad el gráfico de contornos para $T=53$ y a la derecha el gráfico de contornos para $T=61$. El punto de color azul en el gráfico de la izquierda corresponde al valor máximo de $\hat{E}(G S)=0,3546$ que se obtiene con la combinación $T=45, N=3$ y $C=6$.

Tras

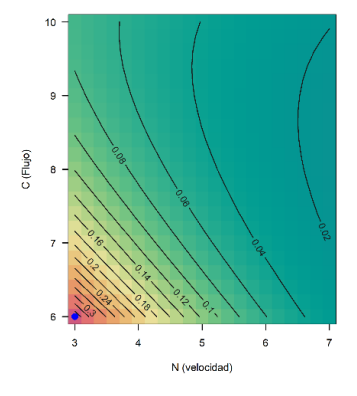

T=53

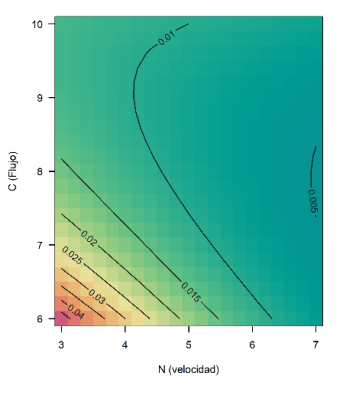

$T=61$

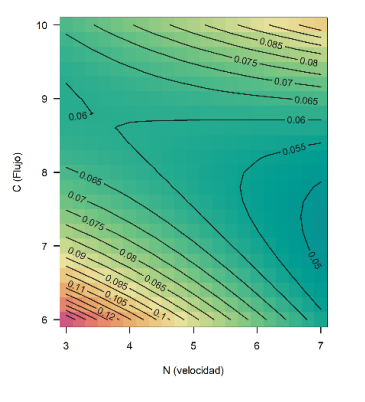

Usando la ecuación de $\widehat{\operatorname{Var}}(G S)$ mostrada anteriormente, se aplicó un método de búsqueda para obtener los valores de $T, N$ y $C$ que minimizan la varianza de variable grado de secado $G S$ y lo que se encontró fue que la menor variabilidad del grado de secado es $1,264844 \mathrm{e}-05$ y se obtiene cuando $T=51,6 ; N=7$ y $C=8$.

En la Figura 6 se muestran los gráficos de contornos para $\widehat{\operatorname{Var}}(G S)$ cuando la variable $T=45 ; 51,6 ; 61$. En el panel central hay un punto de color azul para identificar la combinación $T=51,6 ; N=7$ y $C=8$ que minimiza la varianza estimada de $G S$.

Figura 6. Gráfico de contornos para $\widehat{\operatorname{Var}}(G S)$. En la izquierda el gráfico de contornos para $T=45$, en la mitad el gráfico de contornos para $T=51,6$ y a la derecha el gráfico de contornos para $T=61$. El punto de color azul en el gráfico de la mitad corresponde al valor mínimo de $\widehat{\operatorname{Var}}(G S)=1,264844$ - 05 que se obtiene con la combinación $T=51,6 ; N=7$ y $C=8$.

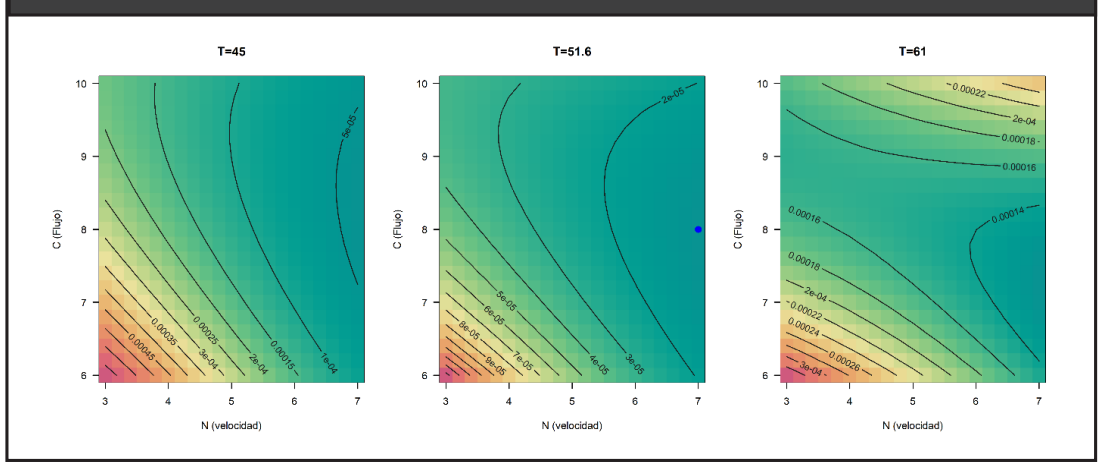




\section{Conclusiones}

En este artículo se logró encontrar un modelo de regresión beta para explicar la variable $G S$ en función de las variables velocidad rotacional del tornillo $(N)$, temperatura $(T)$ y flujo de alimentación $(C)$. Este nuevo modelo presentó unas medidas de desempeño mejores que el modelo inicial propuesto por Triana et al. (2009) para el mismo conjunto de datos. Del modelo propuesto en el presente artículo se destaca la expresión matemática para el valor esperado o promedio del grado de secado $\widehat{E}(G S)$. Esta expresión matemática sirve a los investigadores para predecir el grado de secado cuando las variables experimentales velocidad rotacional del tornillo $(N)$, temperatura $(T)$ y flujo de alimentación $(C)$ se fijan en valores específicos. Adicionalmente, en el artículo se identificó que la combinación $T=45, N=3$ y $C=6$ maximiza el grado de secado a un valor de $35,46 \%$ (o 0,3546 en fracción).

\section{Referencias}

Akaike, H. (1974). A new look at the statistical model identification. IEEE Transactions on Automatic Control, 19(6), pp. 716-723. [Online] Disponible en: http://dx.doi. org/10.1109/TAC.1974.1100705. [Consultado 6 de junio de 2020].

Bai, J.W.; Xiao, H.W.; Ma, H.L.; Zhou, C.S. (2018). Artificial Neural Network Modeling of Drying Kinetics and Color Changes of Ginkgo Biloba Seeds during Microwave Drying Process. J. Food Quality. [Online] Disponible en: https://doi.org/10.1155/2018/3278595. [Consultado 6 de junio de 2020].

Farzad, M.; Yagoobi, J. (2020). Drying of moist cookie doughs with innovative slot jet reattachment nozzle. Drying Technology, in press. [Online] Disponible en: https://doi.org/10. 1080/07373937.2020.1729173. [Consultado 6 de junio de 2020].

Gauss, C.F. (1809). Theoria Motus Corporum Coelestium in Sectionibus Conicis Solem Ambientum.

Jafari, A.; Zare, D. (2017). Ultrasound-assisted fluidized bed drying of paddy: Energy consumption and rice quality aspects, Drying Technology, 35(7), 893-902. [Online] Disponible en: https://doi.org/10.1080/07373937.2016.1230742. [Consultado 6 de junio de 2020].

Jumah, R.Y.; Mujumdar, A.S. (1995). Dryer feeding systems. In Handbook of Industrial Drying, 2nd Ed; Mujumdar, A.S., Ed.; Marcel Dekker, Inc.:New York; Vol. 2, 1151-1178.

Legendre, A. M. (1805). Nouvelles méthodes pour la détermination des orbites des comètes. Paris: Courcier.

Li, K.; Zhang, M.; Mujumdar, A.S.; Chitrakar, B. (2019). Recent developments in physical field-based drying techniques for fruits and vegetables, Drying Technology, 37(15) 1954-1973. [Online] Disponible en: https://doi.org/10.1080/07373937.2018.1546733. [Consultado 6 de junio de 2020].

Mustaffar, A.; Phan, A.; Boodhoo, K. (2018). Hybrid heat pipe screw dryer: A novel, continuous and highly energy-efficient drying technology. Chemical Engineering and Processing - Process Intensification, 128, 199-215. 
Taruna, I.; Jindal, V.K. (2002). DRYING OF SOY PULP (OKARA) IN A BED OF INERT PARTICLES, Drying Technology, 20(4-5), 1035-1051. [Online] Disponible en: https://doi. org/10.1081/DRT-120004012. [Consultado 6 de junio de 2020].

Triana, M.A.; Ayala, M.A.; De la Puente, F.; Camargo-Amado, R. (2009). Grado de secado y eficiencia térmica de un secador de tornillo transportador drying. DYNA, 78(165), pp. 196-206.

R Core Team (2020). R: A language and environment for statistical computing. R Foundation for Statistical Computing, Vienna, Austria. URL: https://www.R-project.org/.

Reyes, A.; Vega, R.; García, G. (2008). Drying Sawdust in a Pulsed Fluidized Bed, Drying Technology, 26(4), 476-486. [Online] Disponible en: https://doi. org/10.1080/07373930801929508. [Consultado 6 de junio de 2020].

Rigby, R.A.; Stasinopoulos, D.M. (2005). Generalized additive models for location scale and shape. Applied Statistics Series C, 54(3), pp. 507-554. [Online] Disponible en: https:// www.jstor.org/stable/3592732. [Consultado 6 de junio de 2020].

Stasinopoulos, M; Rigby, R.; Heller, G.; Voudouris, V.; De Bastiani, F. (2017). Flexible Regression and Smoothing: Using GAMLSS in R. Chapman \& Hall/CRC. 